\title{
Desafios da prevenção do novo coronavírus (COVID-19) na prática odontológica
}

\author{
Challenges of preventing the new coronavirus (COVID-19) in dental practice \\ Desafíos de prevenir el nuevo coronavirus (COVID-19) en la práctica dental
}

\begin{abstract}
RESUMO
OBJETIVO: Descrever os principais desafios enfrentados na prática odontológica acerca dos métodos de prevenção da COVID-19. MÉTODO: Trata-se de uma revisão de literatura, foram utilizados artigos nacionais e internacionais publicados no primeiro semestre de 2020, indexados nas plataformas MEDLINE, LILACS e BBO. Para seleção dos artigos utilizou-se os descritores "COVID-19" e "Odontologia", resultando em uma amostra de 5 artigos. RESULTADOS: Considerando o ambiente de trabalho, as práticas e instrumentos utilizados pelos cirurgiões-dentistas, são profissionais constantemente expostos a riscos biológicos. As medidas de proteção utilizadas antes da pandemia, não são totalmente eficazes para impedir a contaminação por COVID-19, causando apreensão nos profissionais, e a necessidade de adquirirem conhecimento sobre as formas de prevenção e controle da Covid-19. CONCLUSÃO: Os desafios incluem a qualidade das informações sobre a doença, as adaptações relativas à biossegurança, geração de protocolos, o medo e a ansiedade presentes no cotidiano dos profissionais no atual contexto sanitário.

DESCRITORES: Odontologia; COVID-19; Atendimento Integral à Saúde, Biossegurança.
\end{abstract}

\section{ABSTRACT}

Describe the main challenges faced in dental practice about COVID-19 prevention methods. METHOD: This is a literature review, using national and international articles published in the first half of 2020, indexed on the MEDLINE, LILACS and BBO platforms. To select the articles, the descriptors "COVID-19" and "Dentistry" were used, resulting in a sample of 5 articles for complete reading. RESULTS: Considering the work environment, the practices and instruments used by dentists, they are professionals constantly exposed to biological risks. The protective measures used before the pandemic, are not fully effective to prevent contamination by COVID-19, causing apprehension among professionals, and the need to acquire knowledge about the forms of prevention and control of Covid-19. CONCLUSION: The challenges include the quality of information about the disease, adaptations related to biosafety, generation of protocols, fear and anxiety present in the daily lives of professionals in the current health context.

DESCRIPTORS: COVID-19, Dentistry,integrated health care, Biosecurity.

\section{RESUMEN}

Describa los principales desafíos que enfrenta la práctica dental sobre los métodos de prevención de COVID-19. MÉTODO: Esta es una revisión de la literatura, que utiliza artículos nacionales e internacionales publicados en la primera mitad de 2020, indexados en las plataformas MEDLINE, LILACS y BBO. Para seleccionar los artículos, se utilizaron los descriptores "COVID-19" y "Odontología", lo que resultó en una muestra de 5 artículos para lectura completa. RESULTADOS: Considerando el ambiente de trabajo, las prácticas e instrumentos utilizados por los dentistas, son profesionales constantemente expuestos a riesgos biológicos. Las medidas de protección utilizadas antes de la pandemia no son completamente efectivas para prevenir la contaminación por COVID-19, causando aprensión entre los profesionales, y la necesidad de adquirir conocimiento sobre las formas de prevención y control de Covid-19. CONCLUSIÓN: Los desafíos incluyen la calidad de la información sobre la enfermedad, las adaptaciones relacionadas con la bioseguridad, la generación de protocolos, el miedo y la ansiedad presentes en la vida cotidiana de los profesionales en el contexto de salud actual.

DESCRIPTORES: COVID-19, Odontología, atención médica integrada, Biosec.

RECEBIDO EM: 13/08/2020 APROVADO EM: 15/09/2020

\section{Camila Caroline da Silva}

Cirurgiã-Dentista, pós Graduanda em Implantodontia pelo SEC - Schettini Educação Continuada, Recife.

ORICID: 0000-0002-1623-7630 


\section{Danielle Ramalho Barbosa da Silva}

Cirurgiã Dentista, Residente em Odontologia em Saúde Coletiva /SESAU-Recife. ORICID: 0000-0001-72813439

\section{Gutargo Nunes Teixeira}

Cirurgião-Dentista, Residente em Odontologia em Saúde Coletiva /SESAU-Recife. ORICID: 0000-0003-1115-9677

\section{Victor Figuerêdo Sabino de Lima}

Cirurgião-Dentista, Pós-graduando em ortodontia pelo CPGO-Recife. ORICID: 0000-0002-3890-7471

\section{William da Silva Ribeiro}

Enfermeiro, Cirurgião-Dentista especialista em Saúde Pública com Ênfase em Saúde da Família pela UNINTER. ORICID: 000-0001-7290-8972

\section{INTRODUÇÃO}

E m dezembro de 2019, alguns casos de pneumonia foram encontrados em Wuhan, na província de Hubei, China. Esta pneumonia mais tarde foi atribuída a infecção por um novo coronavírus (SAR-CoV-2) e sua manifestação clínica batizada de COVID-19¹ .

Os coronavírus fazem parte de uma família de vírus causadores de infecções respiratórias e a sua nomenclatura advém do seu aspecto microscópico semelhante a espigas em forma de coroa em sua superfície. Espalhando-se por todo o mundo, a COVID-19 recebeu da Organização Mundial de Saúde (OMS) o status de Pandemia, demandando esforços articulados da comunidade internacional ${ }^{2}$.

O curso clínico da doença é heterogêneo, com pacientes apresentando formas assintomáticas enquanto outra parcela evolui para quadros clínicos classificados como leves, moderados ou severos, conforme o grau de comprometimento clínico ${ }^{3}$.

Segundo dados da OMS 4 , o primeiro caso confirmado de COVID-19 no
Brasil foi em 26 de fevereiro de 2020. O Brasil é um dos países mais afetados pela pandemia de COVID-19, em junho de 2020 tornou-se o segundo país com maior número de casos e de óbitos atribuídos à COVID-19.

Diante desta emergência de saúde pública, este estudo, verifica na literatura, os principais desafios enfrentados por cirurgiões-dentistas para adaptar a rotina dos serviços odontológicos na prevenção a COVID-19.

\section{MÉTODOS}

Trata-se de uma revisão integrativa sobre os principais desafios da prevenção do novo coronavírus (COVID-19) enfrentados na prática odontológica. Realizou-se uma busca por artigos publicados nas bases de dados MEDLINE (Sistema Online de Busca e Análise de Literatura Médica), LILACS (Literatura Latino-americana e do Caribe em Ciências da Saúde) e BBO (Bibliografia Brasileira de Odontologia). Para a busca, utilizou-se as palavras-chaves: Covid-19 e Odonto-
Quadro 1: Artigos selecionados nas bases de dados.

\begin{tabular}{|c|c|c|}
\hline BASE DE DADOS & ENCONTRADOS & SELECIONADOS \\
\hline MEDLINE & 86 & 5 \\
\hline LILACS & 23 & 0 \\
\hline BBO & 6 & 0 \\
\hline TOTAL & 109 & 5 \\
\hline Fonte: Dados de pesquisa,2020. & \multicolumn{2}{|}{} \\
\hline
\end{tabular}

logia, utilizando-se o operador booleano AND para combinação.

Foram incluídos artigos em língua inglesa, portuguesa e espanhola, publicados nas bases de dados supracitadas durante o período de 1 de janeiro de 2020 a 30 de junho de 2020. Estudos que atendessem os objetivos deste estudo, com texto completo disponível. Não foram incluídos: estudos relacionados a educação odontológica, teleodontologia/telessaúde, conhecimento dos pacientes, artigos de revisão, overview, resumos, editoriais e guidelines.

$\mathrm{Na}$ busca inicial foram encontrados 109 resultados. Ao considerar os critérios de inclusão e exclusão e em seguida leitura dos resumos, foram selecionados 5 artigos. Esse processo está descrito no quadro 1 . Os dados dos estudos selecionados foram organizados em uma planilha com as seguintes informações: Título, autores, objetivo, resultados, conclusão.

\section{RESULTADOS}

Todos os profissionais de saúde precisaram adaptar suas ações durante a pandemia. Os dentistas se destacam nesse contexto, pois com o uso de equipamentos utilizados constantemente nos procedimentos odontológicos, geram-se gotículas e aerossóis que se espalham no ambiente. Dessa forma, aparelhos odontológicos podem contaminar-se após o uso ou ficarem exposto a um ambiente clínico contaminado. Devido a isto Meng et al.5, afirmam que as medidas padrão de proteção no trabalho clínico di- 
ário não são suficientemente eficazes para impedir a disseminação da doença.

Segundo Botta et al.6, uma das formas de limitar a transmissão aérea de patógenos no ambiente odontológico é inativar os vírus o mais rápido possível após sua produção e bloquear as rotas de transmissão de pessoa para pessoa nas clínicas odontológicas.

Com o aumento do número de casos de Covid-19 no Brasil, os cirurgiões-dentistas devem estar aptos a identificar pacientes com sintomas da doença e adotar condutas preventivas na prática clínica, seguindo as medidas de biossegurança em todos os atendimentos?

Diante do contexto de pandemia de COVID-19 torna-se mais viável realizar atendimentos odontológicos apenas em casos de urgências e emergências. O custo-benefício para realização de procedimentos odontológicos de forma indiscriminada durante a pandemia, pode ser considerado um agravo maior à população, do que um auxílio propriamente dito ${ }^{3}$.

O quadro 2 apresenta os principais resultados encontrados nos artigos selecionados para a amostra do presente estudo.

\section{Quadro 2: Descrição dos dados encontrados nos artigos incluídos.}

\begin{tabular}{|c|c|c|c|c|c|}
\hline No & TíTULO & AUTORES & OBJETIVO & RESULTADOS & CONCLUSÃO \\
\hline 1 & $\begin{array}{c}\text { Conhecimento } \\
\text { sobre o novo coro- } \\
\text { navírus (COVID-19) } \\
\text { entre estagiários, } \\
\text { auxiliares e especia- } \\
\text { listas em odon- } \\
\text { tologia na Arábia } \\
\text { Saudita: um estudo } \\
\text { nacional. }\end{array}$ & $\begin{array}{l}\text { Quadri MFA, } \\
\text { et al. }\end{array}$ & $\begin{array}{l}\text { Investigar o conhe- } \\
\text { cimento atual sobre } \\
\text { o COVID-19 entre os } \\
\text { profissionais de saúde } \\
\text { bucal. }\end{array}$ & $\begin{array}{l}\text { O conhecimento estava signifi- } \\
\text { cativamente relacionado ao nível } \\
\text { de qualificação. No entanto, a } \\
\text { diferença na fonte de informação } \\
\text { não demonstrou nenhum efeito. }\end{array}$ & $\begin{array}{c}\text { O conhecimento básico sobre o } \\
\text { COVID-19 entre os profissionais } \\
\text { de saúde bucal na Arábia Saudita } \\
\text { é aceitável. }\end{array}$ \\
\hline 2 & $\begin{array}{l}\text { Surto de COVID-19 } \\
\text { no norte da Itália: } \\
\text { uma visão geral } \\
\text { sobre odontologia. } \\
\text { Uma pesquisa de } \\
\text { questionário. }\end{array}$ & $\begin{array}{l}\text { Cagetti MG, } \\
\text { et al. }\end{array}$ & $\begin{array}{l}\text { Avaliar os sinais/ } \\
\text { sintomas, medidas } \\
\text { de proteção, } \\
\text { conscientização e } \\
\text { níveis de percepção } \\
\text { em relação ao } \\
\text { COVID-19 entre } \\
\text { dentistas na } \\
\text { Lombardia, Itália. }\end{array}$ & $\begin{array}{l}\text { 14,43\% dos participantes } \\
\text { apresentaram um ou mais } \\
\text { sintomas relacionados a } \\
\text { COVID-19. Trinta e um indivíduos } \\
\text { foram positivos ao vírus } \\
\text { SARS-CoV-2 e } 16 \text { indivíduos } \\
\text { desenvolveram a doença. } 2 \% \text { dos } \\
\text { dentistas estavam confiantes } \\
\text { em evitar a infecção, dentistas } \\
\text { que trabalhavam em áreas de } \\
\text { baixa prevalência de COVID-19 } \\
\text { estavam mais confiantes do que } \\
\text { aqueles que trabalham nas áreas } \\
\text { de alta prevalência. }\end{array}$ & $\begin{array}{c}\text { Esta pesquisa demonstrou } \\
\text { que os dentistas na área de } \\
\text { maior prevalência do COVID-19, } \\
\text { embora relatassem ter mais } \\
\text { sintomas/sinais do que o } \\
\text { restante da amostra, foram os } \\
\text { que adotaram mais medidas } \\
\text { preventivas, embora estivessem } \\
\text { menos confiantes em evitar a } \\
\text { infecção. }\end{array}$ \\
\hline 3 & $\begin{array}{l}\text { Investigação das } \\
\text { atitudes e compor- } \\
\text { tamentos clínicos } \\
\text { dos dentistas } \\
\text { turcos em relação } \\
\text { à pandemia de CO- } \\
\text { VID-19: um estudo } \\
\text { de pesquisa }\end{array}$ & $\begin{array}{l}\text { Duruk G, } \\
\text { Gümüșboğa } \\
\text { ZS e çolak C. }\end{array}$ & $\begin{array}{l}\text { Investigar que tipo } \\
\text { de precauções os } \\
\text { dentistas turcos } \\
\text { tomaram em clínicas } \\
\text { odontológicas durante } \\
\text { a pandemia de CO- } \\
\text { VID-19. }\end{array}$ & $\begin{array}{c}\text { Mais de } 90 \% \text { dos dentistas esta- } \\
\text { vam preocupados com si mes- } \\
\text { mos e suas familias. Apenas } 12 \% \\
\text { dos dentistas usavam máscaras } \\
\text { N95. Embora os dentistas turcos } \\
\text { tenham tomado algumas medi- } \\
\text { das de precaução, não tomaram } \\
\text { precauções suficientes para se } \\
\text { protegerem, proteger profissio- } \\
\text { nais da equipe e pacientes. }\end{array}$ & $\begin{array}{l}\text { É altamente recomendável que } \\
\text { os dentistas tomem as pre- } \\
\text { cauções máximas no ambiente } \\
\text { clínico. As diretrizes sobre a pan- } \\
\text { demia de COVID-19 devem ser } \\
\text { enviadas a todos os dentistas } \\
\text { pelas associações odontológicas } \\
\text { regionais e nacionais. }\end{array}$ \\
\hline 4 & $\begin{array}{l}\text { Odontologia } \\
\text { durante a epidemia } \\
\text { COVID-19: um fluxo } \\
\text { de trabalho italiano } \\
\text { para o gerencia- } \\
\text { mento da prática } \\
\text { odontológica }\end{array}$ & $\begin{array}{l}\text { Peditto M, } \\
\text { et al. }\end{array}$ & $\begin{array}{l}\text { Apresentar um } \\
\text { fluxo de trabalho } \\
\text { de gerenciamento } \\
\text { de procedimentos } \\
\text { odontológicos, já } \\
\text { em uso na Unidade } \\
\text { de Odontologia do } \\
\text { Hospital Universitário } \\
\text { de Messina. }\end{array}$ & $\begin{array}{l}\text { Não foram detectados casos de } \\
\text { infecção por COVID-19 entre pa- } \\
\text { cientes e funcionários da unidade } \\
\text { odontológica em um período de } \\
\text { dois meses enquanto se usava } \\
\text { este protocolo. }\end{array}$ & $\begin{array}{l}\text { Esse fluxo de trabalho parece } \\
\text { uma solução promissora e eficaz } \\
\text { para gerenciar procedimen- } \\
\text { tos odontológicos durante a } \\
\text { epidemia de COVID-19 e pode } \\
\text { ser implementado em práticas } \\
\text { públicas e privadas até que a } \\
\text { emergência seja contida. }\end{array}$ \\
\hline
\end{tabular}




\begin{tabular}{|c|c|c|c|c|c|}
\hline 5 & $\begin{array}{l}\text { Medo e modifi- } \\
\text { cações na prática } \\
\text { entre dentistas para } \\
\text { combater o surto } \\
\text { do novo coronavírus } \\
\text { (COVID-19) }\end{array}$ & $\begin{array}{c}\text { Ahmed MA, } \\
\text { et al. }\end{array}$ & $\begin{array}{l}\text { Avaliar a ansiedade e } \\
\text { medo de se infectar } \\
\text { entre os dentistas } \\
\text { no trabalho duran- } \\
\text { te o surto do novo } \\
\text { coronavírus (CO- } \\
\text { VID-19). Assim como, } \\
\text { o conhecimento dos } \\
\text { dentistas sobre as } \\
\text { várias modificações } \\
\text { práticas para combate } \\
\text { da COVID-19. }\end{array}$ & $\begin{array}{l}\text { 78\% dos clínicos gerais parti- } \\
\text { cipantes, estavam ansiosos } \\
\text { e assustados com os efeitos } \\
\text { devastadores da COVID-19. 90\% } \\
\text { dos dentistas estavam cientes } \\
\text { das mudanças recentes nos } \\
\text { protocolos de tratamento. }\end{array}$ & $\begin{array}{l}\text { Os dentistas estão em um } \\
\text { estado de ansiedade e medo } \\
\text { enquanto trabalham em seus } \\
\text { respectivos campos devido ao } \\
\text { impacto pandêmico da CO- } \\
\text { VID-19 na humanidade. Várias } \\
\text { práticas odontológicas foram } \\
\text { modificadas de acordo com as } \\
\text { diretrizes, sendo recomendados } \\
\text { apenas tratamento de emer- } \\
\text { gência ou o encerramento das } \\
\text { práticas por um período incerto. }\end{array}$ \\
\hline
\end{tabular}

\section{DISCUSSÃO}

Ao tomar conhecimento do surgimento de uma nova doença, o primeiro desafio para os profissionais é a qualidade da informação que acessam. Não só no tocante às medidas preventivas, mas também, para saber informar corretamente seus pacientes. Em Duruk et al. ${ }^{8}$, a principal fonte de informação dos cirurgiões-dentistas participantes da pesquisa foram sites e contas de mídia social, só $26,65 \%$ dos profissionais participantes compareceram a reuniões informativas sobre COVID-19.

Em Quadri et al. ${ }^{9}$, apesar dos participantes da pesquisa que afirmaram ter recebido orientações prévias sobre $\mathrm{CO}$ VID-19, relatarem fazer uso de fontes confiáveis. Ainda haviam equívocos entre as informações, os mesmos erros foram encontrados no grupo que afirmou não ter recebido orientações prévias. Neste último grupo, observou-se após a intervenção, que o número de participantes com respostas corretas para a maioria das questões de conhecimento aumentou significativamente.

Nesse contexto também é importante atentar-se à saúde mental dos profissionais. Em Ahmed et al. ${ }^{10}$, grande parte dos profissionais que participaram da pesquisa, tinham medo de se infectarem com COVID-19 de um paciente ou colega de trabalho, se sentiam ansiosos ao tratar um paciente que apresentasse tosse ou que estivesse com suspeita de infecção por COVID-19, e também se sentiram nervosos ao conversar com pacientes proximamente.
Isso reforça a importância da gestão e representantes da categoria ouvirem e assistirem os profissionais em suas dúvidas, ansiedades e medos, criarem protocolos e cursos online, para capacitar toda a equipe de saúde bucal, orientar funcionários e pacientes, entre outras estratégias pertinentes e viáveis.

Durante a utilização do fluxo de trabalho apresentado em Peditto et al. ${ }^{11}$, nenhum novo caso COVID-19 foi detectado entre pacientes e funcionários da Unidade de Odontologia do Hospital Universitário de Messina. Uma das principais medidas apresentadas foi o gerenciamento remoto. A unidade conseguiu gerenciar até 20 pacientes remotamente e 12 pacientes pessoalmente usando o fluxo de trabalho proposto por dia útil, das $8 \mathrm{~h}$ às $14 \mathrm{~h}$.

Em Cagetti et al. ${ }^{12}$, os dentistas que trabalharam durante a pandemia adotaram várias medidas de prevenção antes $\mathrm{da}$ chegada do paciente (como espaço entre as consultas para não saturar a sala de espera e triagem por telefone), na sala de espera (ventilação frequente e verificar o status de saúde atual do paciente) e na sala de operações (lavar as mãos antes e depois de cada procedimento, remoção de equipamentos de proteção descartáveis e desinfecção de equipamentos, ventilação da área de operação por pelo menos 10 minutos após cada paciente).

Algumas dessas medidas diminuem a quantidade de procedimentos odontológicos realizados no dia, pois gera mais tempo para as adaptaçôes, mas vale a pena para atender em um ambiente mais seguro 
para os profissionais e os pacientes. Outras práticas mencionadas já deviam ser aplicadas de forma rotineira, pois, não é apenas durante a pandemia que os dentistas estão expostos a riscos biológicos em seu ambiente de trabalho. Apesar dos desafios impostos, espera-se que após esse período, os profissionais seguirão mais atentos às normas de biossegurança.

\section{CONCLUSÃO}

Conclui-se que, assim como os demais profissionais de saúde, os cirurgiões dentistas precisaram adequar as suas ações durante a pandemia, destacando-se o fato destes estarem constantemente expostos a aerossóis provenientes de sua prática clínica, o que lhes confere grande risco de contaminação. Os desafios incluem a qualidade das informações sobre a doença, as adaptações relativas à biossegurança, geração de protocolos, o medo e a ansiedade gerados por uma doença surgida recentemente que ganhou grandes proporções. Governos, órgão de classe e demais autoridades de saúde supranacionais devem reforçar a produção de conteúdo a respeito do novo coronavírus, estando atentos a estratégias comunicacionais capazes de atingir o público alvo, a equipe odontológica. Proporcionando-lhe maior nível de segurança pessoal para tomadas de decisão e para o desempenho de suas atividades profissionais no atual contexto sanitário.

\section{REFERÊNCIAS}

1. Zhang XH, Ling JQ. Guidelines on the prevention and control of disease in dental practice during the coronavirus outbreak. Chin J Dent Res. [Internet]. 2020 [acesso em 12 jul 2020]; 23 (2): 8994. Disponível em: https://www.doi.org/10.3290/j.cjdr.a44743

2. Pereira LJ, Pereira CV, Murata RM, Pardi V, Pereira-Dourado SM. Biological and social aspects of Coronavirus Disease 2019 (COVID-19) related to oral health. Braz Oral Res. [Internet]. 2020 [acesso em 12 jul 2020]; 34: 1-11. Disponivel em: $\quad$ https://www.scielo.br/scielo.php?script=sci_arttext\&pid=S1806-83242020000100600

3. Franco JB, Camargo AR, Peres MPSM. Cuidados Odontológicos na era do COVID-19: recomendações para procedimentos odontológicos e profissionais. Rev assoc paul cir dent. [Internet]. 2020 [acesso em 12 jul 2020]; 74 (1): 18-21. Disponivel em: http://www.crosp.org.br/uploads/arquivo/8b9e5bd8d0d5fd9cf5f79f81e6cb0e56.pdf

4. World Health Organization. Coronavirus disease (COVID-19) Situation Dashboard. [Internet]. 2020 [acesso em 12 jul 2020]. Disponivel em: https://covid19.who.int/region/amro/country/br

5. Meng L, Hua F, Bian Z. Coronavirus Disease 2019 (COVID-19): Emerging and Future Challenges for Dental and Oral Medicine. J Dent Res. [Internet]. 2020 [acesso em 12 jul 2020]; 95 (5): 481-487. Disponivel em: https://pubmed.ncbi.nlm.nih. gov/32162995/

6. Botta SB, Teixeira FS, Hanashiro FS, Araújo WWR, Cassoni A, Salvadori MCBS. Ultraviolet-C decontamination of a dental clinic setting: required dose and time of UVC light. Braz Dent Sci. [Internet]. 2020 [acesso em 12 jul 2020]; 23 (2): 1-10. Disponível em: https://ojs.ict.unesp.br/index.php/cob/article/view/2275

7. Oliveira JJM, Soares KM, Andrade KS, Farias MF, Romão TCM, Pinheiro RCQ, et al. O impacto do coronavírus (covid-19) na práti- ca odontológica: desafios e métodos de prevenção. Rev Elet Acer Saúde. [Internet]. 2020 [acesso em 12 jul 2020]; 46: 1-12. Disponivel em: https://acervomais.com.br/index.php/saude/article/ view/3487

8. Duruk G, Gümüșboğa ZS, Çolak C. Investigation of Turkish dentists' clinical attitudes and behaviors towards the COVID-19 pandemic: a survey study. Braz Oral Res. [Internet]. 2020 [acesso em 12 jul 2020]; 34: 1-12. Disponível em: https://www.scielo. br/pdf/bor/v34/1807-3107-bor-34-e054.pdf

9. Quadri MFA, Jafer MA, Alqahtani AS, Mutahar SABA, Odabi NI, Daghriri AA, et al. Novel corona virus disease (COVID-19) awareness among the dental interns, dental auxiliaries and dental specialists in Saudi Arabia: A nationwide study. J Infect Public Health. [Internet]. 2020 [acesso em 12 jul 2020]; 13(6): 856-864. Disponivel em: https://www.sciencedirect.com/science/article/pii/ S1876034120304767

10. Ahmed MA, Jouhar R, Ahmed N, Adnan S, Aftab M, Zafar MS, et al. Fear and Practice Modifications among Dentists to Combat Novel Coronavirus Disease (COVID-19) Outbreak. Int J Environ Res Public Health. [Internet]. 2020 [acesso em 12 jul 2020]; 17:1-11. Disponivel em: https://www.mdpi.com/16604601/17/8/2821

11. Peditto M, Scapellato S, Marcianò A, Costa P, Oteri G. Dentistry during the COVID-19 Epidemic: An Italian Workflow for the Management of Dental Practice. Int J Environ Res Public Health. [Internet]. 2020 [acesso em 12 jul 2020]; 17:1-15. Disponivel em: https://pubmed.ncbi.nlm.nih.gov/32403248/

12. Cagetti MG, Cairoli JL, Senna A, Campus G. COVID-19 Outbreak in North Italy: An Overview on Dentistry. A Questionnaire Survey. Int J Environ Res Public Health. [Internet]. 2020 [acesso em 12 jul 2020]; 17 (11): 1-12. Disponível em: https://pubmed. ncbi.nlm.nih.gov/32481672/ 